\title{
Inferring social activities with mobile sensor networks
}

\author{
Trinh Minh Tri Do \\ Idiap Research Institute \\ Switzerland \\ do@idiap.ch
}

\author{
Kyriaki Kalimeri \\ Fondazione Bruno Kessler \\ (FBK-irst) \\ kalimeri@fbk.eu
}

\author{
Bruno Lepri \\ Fondazione Bruno Kessler \\ (FBK-irst) \\ lepri@fbk.eu
}

\author{
Fabio Pianesi \\ Fondazione Bruno Kessler \\ (FBK-irst) \\ pianesi@fbk.eu
}

\author{
Daniel Gatica-Perez \\ Idiap Research Institute \\ EPF Lausanne, Switzerland \\ gatica@idiap.ch
}

\begin{abstract}
While our daily activities usually involve interactions with others, the state-of-the-art methods on activity recognition do not exploit the relationship between social interactions and human activity. This paper addresses the problem of interpreting social activity from human-human interactions captured by mobile sensing networks. Our first goal is to discover different social activities such as chatting with friends from human-human interaction logs and then characterize them by the set of people involved, time and location of the occurring event. Our second goal is to perform automatic labeling of the discovered activities using predefined semantic labels such as coffee breaks, weekly meetings, or random discussions. Our analysis was conducted on interaction networks sensed with Bluetooth and infrared sensors by about fifty subjects who carried sociometric badges over 6 weeks. We show that the proposed system reliably recognized coffee breaks with $99 \%$ accuracy, while weekly meetings were recognized with $88 \%$ accuracy.
\end{abstract}

\section{Categories and Subject Descriptors}

H.4 [Information Systems Applications]: Miscellaneous; D.2.8 [Software Engineering]: Metrics—complexity measures, performance measures

\section{General Terms}

Human Factors

\section{Keywords}

face-to-face interaction, mobile sensing, social activity

\section{INTRODUCTION}

Most of our daily activities involve interactions with others as humans are social by nature. Besides the relationship between human activity and location and time [24], movement and action [4], it also relates to some form of interactions such as having lunch

Permission to make digital or hard copies of all or part of this work for personal or classroom use is granted without fee provided that copies are not made or distributed for profit or commercial advantage and that copies bear this notice and the full citation on the first page. To copy otherwise, to republish, to post on servers or to redistribute to lists, requires prior specific permission and/or a fee.

ICMI'13 Sydney, Australia

Copyright 20XX ACM X-XXXXX-XX-X/XX/XX ...\$15.00. with colleagues, dining with friends, traveling with family. Yet, the relationship between human activity and human-human interactions has not been thoroughly explored.

The challenge of relating social interaction to activity comes from the nature of collected interaction data. Online social, communication, and webpage networks are examples of large-scale interaction networks which have advanced the state of the art in social network analysis $[2,16,3]$. However, the analysis on these data is usually limited to static, aggregated patterns since these networks do not correspond well to in-person interactions in daily life. Interestingly, the introduction of people-centric sensing [6] has open the opportunity to collect and analyze daily social interaction, with various sensing methods such as Bluetooth proximity or WiFi [13].

Bluetooth has been used as a platform for social interaction and communication among groups of users [17]. By analyzing Bluetooth proximity network, a recently proposed instance of topic model, called GroupUs, was able to discover multiple group activities from timestamped social interaction links [11]. While the discovered activity topics were reported to be relatively relevant and can be interpreted by experts, the automatic transformation from discovered activity topics to general semantic meanings (e.g., meeting, chatting, eating) is still missing.

This work was inspired from the above seminal work and our first goal is to generalize the idea to the case of multimodal interaction data. Specifically, our study was conducted on data collected with Bluetooth (BT) and infrared sensors (IR), where interaction links were localized by their proximity to fixed stations. Our research questions for this goal were then closely related to typical multimodal research questions: (a) How to exploit multiple types of interaction sensors, in particular BT and IR sensors?, (b) How to use location data to enhance the social interaction patterns?, and (c) How well does the method perform on this multimodal dataset?.

The second goal of this paper was to build a fully automatic system which senses social interactions, discovers emerging activity topics, then labels the discovered activity topics with a set of predefined activity labels. We believe that such a system has potential applications in several fields such as context-aware applications (e.g., change ringtone when the user enters a meeting) or individual and social behavior analysis (e.g., the dependency between social activity and mood). To achieve this, we proposed a supervised learning framework where activity topics were represented as feature vectors and random forest was used to learn and predict if a given activity topic corresponded to a specific activity label.

This paper makes three major contributions. First, we extended an existing algorithm to handle spatio-temporal context of interactions and allowed the model to work with both BT and IR data. Second, we introduced an automatic labeling method for the set 
of discovered activity topics. This component allows us to have a fully automatic framework for sensing and interpreting humanhuman interaction. Finally, our paper presents a case study of social interactions in a real organization, in which we provided detailed analysis on sensing quality and how well actual activities could be recognized.

The rest of the paper is organized as follows. Section 2 discusses related work in the context of human activity and social interaction analysis. Section 3 presents the corpus on which our analysis were conducted. Then, the proposed extension of GroupUs algorithm is presented in Section 4. Section 5 presents our findings on manual interpretation and automatic labeling of discovered activity topics. Finally, Section 6 provides concluding remarks.

\section{RELATED WORK}

Research on human activity has been focused on individual activity inferred from a variety of data types such as video and audio, accelerometer, and indoor location [25, 8, 19]. While the number of sensors for capturing individual activity keeps increasing over time, another direction is to exploit social media for studying human activity. For example, Noulas et al. collected check-in data from Foursquare, a location-based social network which connects online social networks with physical world, and show that human activity varies withIn the course of a day and of a week [21]. Using data from Twitter, Golder and Macy revealed the dependencies between mood and physical/social activities [14].

Our study on the relationship between human activity and social interactions was inspired from an emerging body of work that is investigating the possibilities of analyzing human and social behavior using mobile sensors. At present, Bluetooth and Wi-Fi networks allow the collection of data on specific structural and temporal aspects of social interaction, offering ways to approximate social interaction as spatial proximity or as the co-location of wearable devices, e.g., by means of Bluetooth hits [20, 1, 12]. These means, however, do not always yield good proxies to the social interactions occurring between the individuals carrying the devices. Mobile phone traces suffer the same problem: they can be used to model human mobility [15] with the great advantage of easily scaling up to millions of individuals; they too, however, offer only rough approximations to social interaction in terms of spatial co-location. Cattuto et al. [7] proposed a framework for monitoring social interactions that reconciles scalability and resolution by means of a sensing tier consisting of inexpensive and unobtrusive active RFID devices. The devices are capable of sensing face-toface interactions of individuals as well as spatial proximity over different length scales down to one meter or less. Another strategy for behavioral data collection is to resort to image and video processing based on cameras placed in the environment [9]. This approach provides very rich datasets that are, in turn, computationally very complex: they require line-of-sight access to the monitored spaces and people, specific effort for equipping the relevant physical spaces and can hardly cope with large scales of data. By using sociometric badges, some previous studies revealed important insights into organizational processes, such as the impact of electronic communications on the business performance of teams [23] or the relationship between several behavioral features captured by sociometric badges, employee self-perceptions (from surveys) and productivity [22].

Finally, unsupervised learning approach has been used for human activity discovery in the past, both for individual activities and social activities. Vahdatpour et al. [26] find recurrent patterns from multidimensional time-series given by multiple wearable sensors. In a few recent papers, we proposed several topic models for captur- ing group interaction patterns from Bluetooth proximity networks $[10,11]$. While these previous studies focus on activity discovery, this paper also considers the automatic labeling task for the discovered activities.

\section{SOCIOMETRIC BADGES CORPUS}

The aim of our study is to investigate the behavioral patterns within organizational environments. The SocioMetric Badges Corpus [18], which is exploited in this work, is a multimodal corpus which has been collected in a research institute for over a six week consecutive period, involving a population of 54 subjects (46 subjects that belong to four computer science research groups and 7 subjects of the IT department), during their working hours. The Sociometric Badge sensors were employed for this study; these sensors are equipped with accelerometers, microphones, bluetooth and infrared sensors which capture: the body movements, prosodic speech features, collocation and face-to-face interactions respectively [23]. For the purposes of our study we have exploited the data provided from the Infrared and Bluetooth sensors.

\subsection{Data collection}

Organizational Information. The subjects involved in the study take part in two distinct categories of employees; the administrative group and the research units. The population of the study consisted of 54 subjects, males $=90.8 \%$, females $=9.2 \%$ with a mean of 36.83 years of age and standard deviation of 8.61 years. Due to technical issues, the Bluetooth data was missing for 5 subjects. This study is then conducted on the data of 49 subjects.

Bluetooth Data. Bluetooth detections can be used as a coarse indicator of proximity between devices. Radio signal strength indicator (RSSI) is a measure of the signal strength between transmitting and receiving devices. The range of RSSI values for the radio transceiver in the badge is $(-128,127)$. All sociometric badges broadcast their ID every five seconds using a $2.4 \mathrm{GHz}$ transceiver ( $T R_{\text {radio }}=12$ transmissions per minute). Figure 1(a) shows the Bluetooth proximity network where nodes correspond to subjects (colored by office number) and the strengths of ties correspond to the number of Bluetooth hits. The sensed network is relatively dense and highly affected by the locations of subjects' office. This finding is not surprising given that the physical range of Bluetooth is around 10 meters (Class 2), meaning that a large proportion of BT hits do not correspond to an actual face-to-face interaction.

Infrared data. Infrared transmissions are used to detect faceto-face interactions between people. In order for a badge to be detected through an IR sensor, two individuals must have a direct line of sight and the receiving badge's IR must be within the transmitting badge's IR signal cone of height $h \leq 1$ meter and a radius of $r \leq h \tan \theta$, where $\theta= \pm 15^{\circ}$ degrees. The infrared transmission rate $\left(T R_{i r}\right)$ was set to $1 \mathrm{~Hz}$. The accumulated IR network is visualized in Figure 1(c) in the same way as the one for the BT network. Since IR hits corresponds well to face-to-face interactions, the IR network is less influenced by desk locations. Its structure is then more complex than the BT networks.

Localizing interaction data. Seventeen BT devices were used as fixed stations at key locations in order to infer the location of subjects and their interactions. These points were the restaurant, the cafeteria and the coffee machines as well the meeting and seminar rooms at the hosting organization. The BT devices used for localization have been grouped in four broader categories meeting rooms, admin meeting room, restaurant, and cafeteria. For each BT or IR hit (interaction hit), we find the nearest BT hit between 


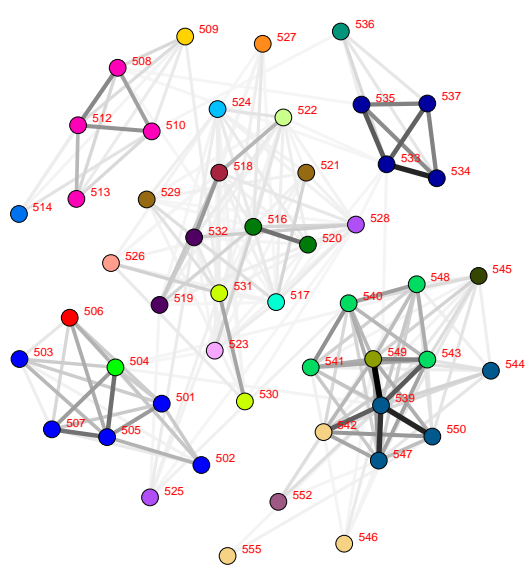

(a) Raw Bluetooth hits

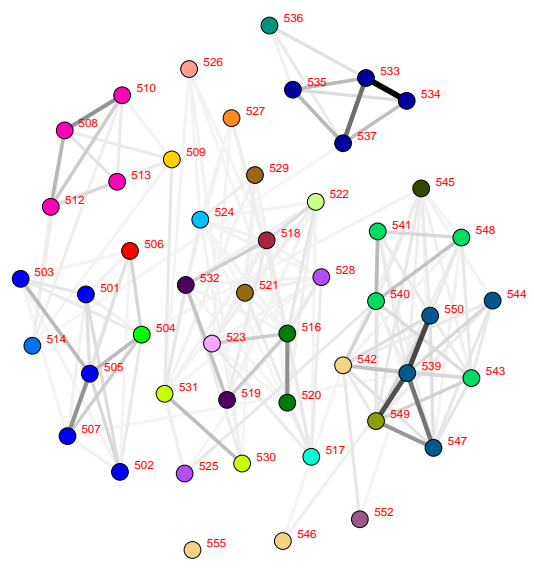

(b) Strong Bluetooth hits

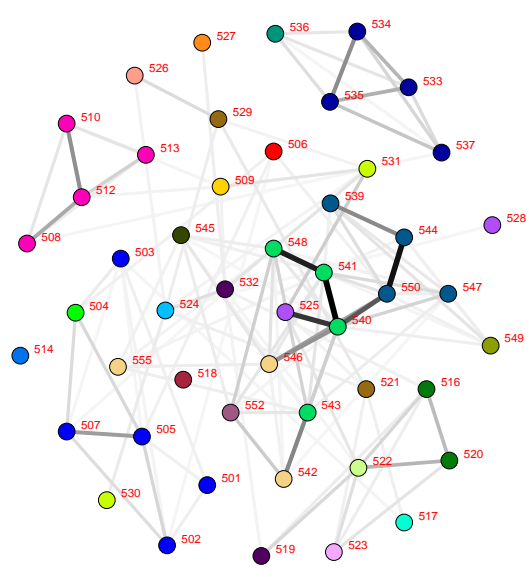

(c) Infrared hits

Figure 1: In-person interaction networks captured by Bluetooth and Infrared sensors. Nodes are colored by the office numbers.

the observer and one of the fixed stations (localization hit), then added the found location to the interaction hit if the time difference between two hits is less than one minute. At the end, the locations of BT or IR hits among subjects belong to one of the four categories above or a special category called others in case where there is no BT hit to any fixed station during the interaction.

\subsection{BT or IR for interaction sensing?}

While IR hits imply actual interaction between two people, the strict detection conditions (of a direct line of sight and angles) mean that the device may fail to capture actual interaction in several situations such as group meeting (e.g., people sit around a big table) or when two interlocutors look at the same object (e.g., screen, board). In practice, we found that IR data is very sparse and its density is about $2 \%$ of the BT data set.

As an alternative to IR, BT proximity can be used as a reliable method to sense face-to-face interaction with low false negative rate. When using Bluetooth proximity data, the challenge is how to reduce its high false positive detection rate which comes from its relatively long range compared to the face-to-face interaction.

With these discussions in mind, we chose to combine both IR and BT data, for which we only keep BT hits with strong signal strength (high RSSI value). In our experiment, a RSSI value greater or equal than -80 is considered as strong, which is generally produced when the distance of the two devices is less than 5 meters (which is sufficient to detect interactions among people in a meeting room of medium size). Figure 2 shows the distributions of RSSI values of BT hits when there is an IR hit (that is, when there is a face-to-face interaction) and when there is not (i.e., we are not sure if there is a real interaction). For the given threshold, the plot shows that $88 \%$ of IR hits can be captured by strong BT hits, and $24 \%$ of BT hits were classified as strong. The accumulated network constructing from strong BT hits is showed in Figure 1(b).

\section{SOCIAL ACTIVITY DISCOVERY WITH TOPIC MODEL}

Topic models are widely used for text analysis to find different topics from text corpora and to summarize documents based on the set of addressed topics. For example, latent Dirichlet allocation (LDA) takes as input a corpus of documents, represented as bags of words, and outputs the set of discovered topics, characterized by their most frequent words [5]. In LDA, each word is assumed

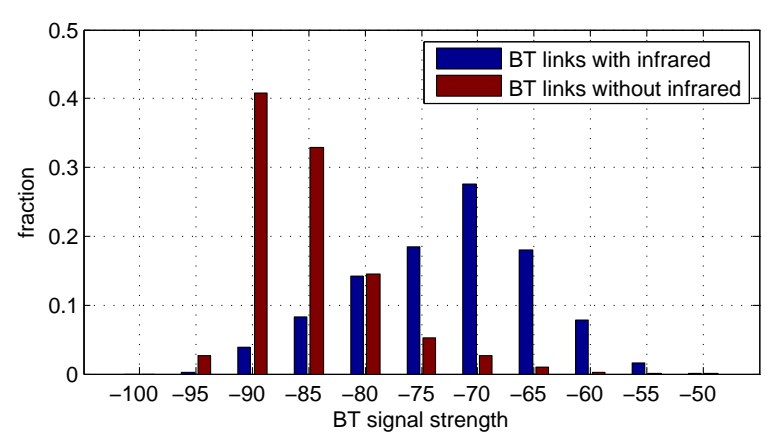

Figure 2: BT signal strength distributions change significantly depending on whether the face-to-face interaction is captured by IR or not.

to be generated from a (latent) topic, and each document can be summarized by a multinomial distribution over topics.

The LDA framework can be applied to human-human interaction mining where a word is an interaction between two individuals and a document consists of interactions captured within a time interval, as in the GroupUs algorithm [11]. While this algorithm works on BT links with temporal context, we extend it to handle spatiotemporal links sensed with BT and IR.

Data representation. The recording period is divided into time slice of $W$ minutes, where $W$ should be large enough so that faceto-face interactions can be captured reliably by at least BT or IR. Each link $i$ contains the following attributes:

$-u_{i}$ : the head of the link (observer)

- $v_{i}$ : the head of the link (observed person)

- $d_{i}$ : day-of-week when the link was observed.

- $h_{i}$ : time-of-day when the link was observed.

$-l_{i}$ : the location where the link was observed.

- $q_{i}$ : the type of the link (BT or IR).

- $s_{i}$ : the identifier of the time slice that the link belongs to. $s_{i} \in$ $\{1 . . S\}$ where $S$ is the total number of time slices.

In the data, we had $d_{i} \in\{$ Mon $, \ldots, F r i\}$ and $h_{i} \in\{8 a m . .6 p m\}$ according to the data collection setting. The location variable can be one of the five location categories described in Section 3.

Probabilistic model. Our extended GroupUs model is illustrated in Figure 3 where shaded nodes correspond to observed vari- 


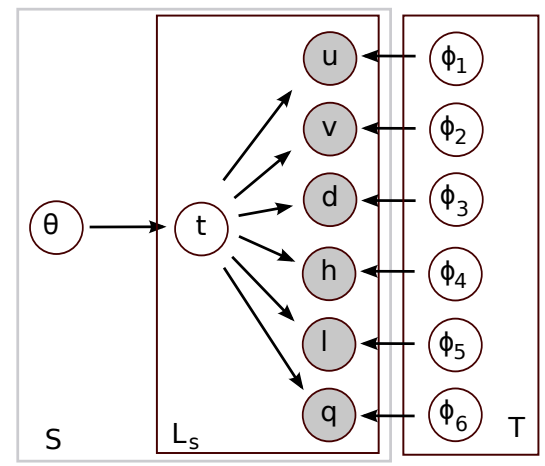

Figure 3: Graphical model of the extended GroupUs for handling spatio-temporal context and other additional attributes of interaction links.

ables, latent variables $t$ correspond hidden activity topics of links, and $\theta$ and $\phi$ are model parameters. We use a plate representation where each node corresponds to a set of random variables, whose size is given by the capital letter in the corner. $S$ stands for the number of slices, $L_{s}$ stands for the number of links in slice $s$, and $T$ is the number of activity topics. Compared to GroupUs, the proposed model has similar structure but contains more observed variables (location $l$ and data type $q$ ), its generative process is then similar to the original algorithm:

\section{Initialization:}

Draw distribution $\theta_{s} \sim \operatorname{Dirichlet}(\boldsymbol{\alpha})$ for each slice $s$.

Draw distribution $\phi_{t} \sim \operatorname{Dirichlet}(\boldsymbol{\beta})$ for each activity topic $t$.

For each link of the slice $s$ :

Draw an activity topic $t \mid s \sim \operatorname{Multinomial}\left(\theta_{s}\right)$.

Draw a first person $u \mid t \sim \operatorname{Multinomial}\left(\phi_{1 t}\right)$.

Draw a second person $v \mid t \sim \operatorname{Multinomial}\left(\phi_{2 t}\right)$.

Draw a day of week $d \mid t \sim \operatorname{Multinomial}\left(\phi_{3 t}\right)$.

Draw a time of day $h \mid t \sim \operatorname{Multinomial}\left(\phi_{4 t}\right)$.

Draw a location $l \mid t \sim \operatorname{Multinomial}\left(\phi_{5 t}\right)$.

Draw a data type $q \mid t \sim \operatorname{Binomial}\left(\phi_{6 t}\right)$.

The model parameter $\phi$ is key for the interpretation of activity topics as it encodes the conditional distributions of observation given activity topics. $\phi_{1 t}$ and $\phi_{2 t}$ characterize who were active members of the group activity corresponding to activity topic $t$. $\phi_{3 t}$ and $\phi_{4 t}$ reveal when the activity happened while $\phi_{5 t}$ indicate where the activity was usually observed. Finally, $\phi_{6 t}$ indicates which type of link is usually observed in the activity topic $t$. The joint probability of observed and unobserved variables can be written by:

$$
\begin{aligned}
& P(\mathbf{u}, \mathbf{v}, \mathbf{d}, \mathbf{h}, \mathbf{l}, \mathbf{q}, \mathbf{s}, \mathbf{t} ; \boldsymbol{\alpha}, \boldsymbol{\beta}) \\
& =\int_{\theta, \phi} P(\mathbf{u}, \mathbf{v}, \mathbf{d}, \mathbf{h}, \mathbf{l}, \mathbf{q}, \mathbf{s}, \mathbf{t}, \theta, \phi ; \boldsymbol{\alpha}, \boldsymbol{\beta}) \partial \theta \partial \phi \\
& =\int_{\theta} P(\mathbf{t} \mid \theta) P(\theta ; \boldsymbol{\alpha}) \partial \theta \int_{\phi} P(\mathbf{u}, \mathbf{v}, \mathbf{d}, \mathbf{h}, \mathbf{l}, \mathbf{q} \mid \mathbf{t}, \phi) P(\phi ; \boldsymbol{\beta}) \partial \phi
\end{aligned}
$$

where the integration over model parameters $\theta$ and $\phi$ can be computed efficiently since we use conjugate priors all link attributes. The model can be learned by collapsed Gibb sampling which samples the posterior distribution $P(\mathbf{t} \mid \mathbf{u}, \mathbf{v}, \mathbf{d}, \mathbf{h}, \mathbf{l}, \mathbf{q} \boldsymbol{\alpha}, \boldsymbol{\beta})$ and provides estimates of $\theta$ and $\phi$.

The output of extended GroupUs algorithm consists of the learned parameters $(\theta$ and $\phi)$ and the vector of topic assignment $\mathbf{t}$. As discussed earlier, each activity topic $t$ can be characterized by the corresponding distributions over people (parameterized by $\phi_{1 t}$ and $\left.\phi_{2 t}\right)$, time $\left(\phi_{3 t}\right.$ and $\left.\phi_{4 t}\right)$, locations $\left(\phi_{5 t}\right)$, and link types $\left(\phi_{6 t}\right)$. However, the topic assignment $\mathbf{t}$ is also helpful for interpreting the discovered activity topics. Basically, this vector $\mathbf{t}$ assigns each link in the dataset to a discovered activity topic, so that we know exactly

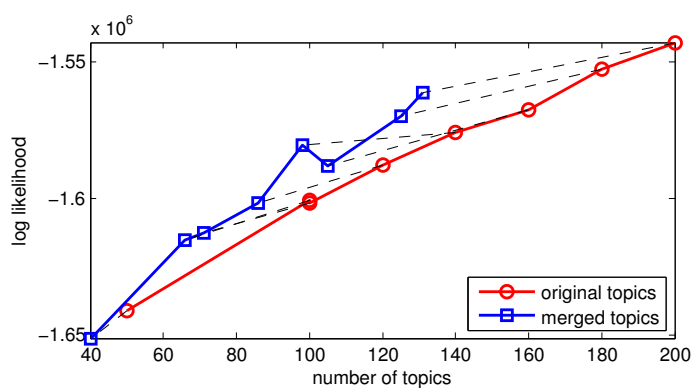

Figure 4: Log likelihood as a function of number of activity topics. Dashed line segments connect results before and after merging. The merging step reduce significantly the number of topics while keeping likelihood value reasonable compared to the original model.

the set links of a given activity topic $t$. Based on this, we can extract additional information about the activity topic such as to visualize the "activeness" of the activity topic over time, find who were involved at a specific time, or to compute the duration of the activity at a specific time.

Post-processing of discovered activity topics. One limitation of GroupUs and topic models in general lies in the number of activity topics $T$ which need to be specified. A too small value of $T$ results in topics which corresponds to multiple activities, and a large value of $T$ wil produce some topics with similar patterns.

We remark that while a person might have multiple activities with another person, activities usually differ in sets of involved people and locations. We thus propose a post-processing process which merge similar activity topics based on who is involved in the activity and where the observed interactions took place. Interestingly, the GroupUs algorithm provided an efficient way to extract the set of active members from the distribution over people, $\phi_{1 t}$ and $\phi_{2 t}$ [11]. For the location, we can simply use the most likely location $\phi_{5 t}$ to define the dominant location of the activity topic.

After the merging step based on active members and dominant location, the model structure is unchanged but the number of topics may be reduced. The sampled topic assignment $\mathbf{t}$ can be transformed to the merged model based on the mapping from the set of original topics to the set of merged topics. Then, the model parameter $\theta$ and $\phi$ of the merged model can be estimated based on the topic assignment according to the merged model.

\section{RESULTS}

We set the slice duration $W$ to 5 minutes, resulting in 3670 non empty slices for the 6 weeks of data. Overall, there are 160,000 dyadic links where the distribution links over 5 location categories meeting rooms, admin meeting room, restaurant,cafeteria, and others are $14.7 \%, 10.8 \%, 7.6 \%, 8.6 \%$, and $58.2 \%$ respectively. We tried multiple values for the number of topic $T$, varying from 50 to 200 , to study the behavior of the model with respect to this hyper parameter. For each value of $T$, the algorithm outputs a set of original activity topics and a corresponding set of merged ones.

Figure 4 shows the log likelihood of the data as a function of the number of topics $T$. The dashed line segments connect results before and after merging topic. As can be seen, the merging step reduces significantly the number of activity topics while keeping the likelihood at a reasonable level. Since the merging step is quite efficient, we continue the study with merged activity topics instead of the original ones. The main advantage is to reduce the number 


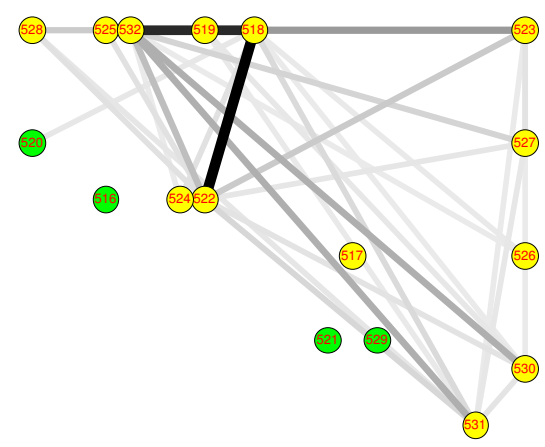

(a) Network visualization
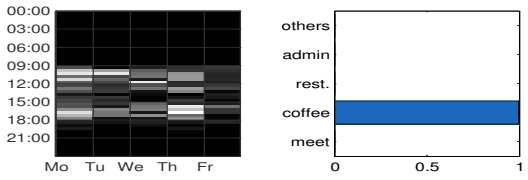

(b) time and location

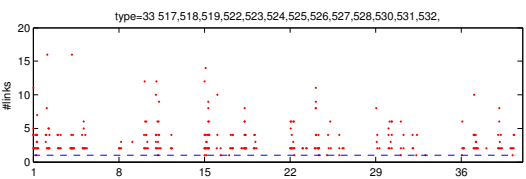

(c) number of link over time (days)
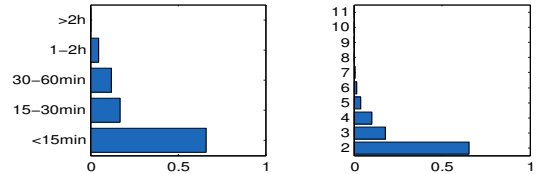

(d) duration and number of people

Figure 5: Example of activity topic visualization for the annotation process. Various information such as time, location, and duration are shown for activity topic 33 in set 3 of the experiment.

of activity topics to be annotated.

The remaining part of this section is dedicated to the interpretation of the discovered topic. In Subsection 5.1, we provide an subjective validation of the set of discovered topics and highlight our findings. In Subsection 5.2, we go further by studying the possibility of automatic interpretation of the set of discovered topics into predefined categories, thus providing a fully automatic framework for human-human interaction sensing.

\subsection{Manual interpretation}

The goal of manual interpretation is to validate if the discovered activity topics are meaningful and to understand which kind of activity can be captured by the extended GroupUs algorithm. We got the annotation done for 6 sets of merged activity topics, which were outputted by the extended GroupUs algorithm with various settings of $T$. The 6 sets consists of 40,66,86, 131, and 71 merged topics and they were numbered from 3 to 8 respectively (Cf. Table 1).

Annotation process: Three people from the organization where the data were collected had accepted to participate in the annotation experiment. Therefore, these annotators are assumed to personally know the subjects, their roles, relationships between them, and also main activities in the organization.

The visualization of each activity topic is built from the outputs of the extended GroupUs algorithm as in Figure 5. Each topic can be described directly with the learned parameter $\phi$, which contains the distribution of links over people, location, and time. We do not include the distribution over link types (BT or IR) these distribution usually is dominated by BT and the patterns are unclear. Besides the plots coming from $\phi$, we also use the assignment of each interaction link to an activity topic (the vector $\mathbf{t}$ ) for more complex visualization such as computing the number of interactions between two given participants for a given activity topic. At the end, there are six different plots for each activity topic $t$ which help the annotator to recognize the activity(ies):

$\diamond$ Interaction network: The network of people for the considered activity topic; active members are highlighted in a different color (yellow). Nodes' positions correspond to the actual desk locations of subjects to simplify the identification of subject.

$\diamond$ Time: The distribution of links over the weekly calendar. While this can be given by the learned parameter $\phi$ in an factorized form $\left(\phi_{3 t} \phi_{4 t}^{\top}\right)$, we decide to estimate the weekly calendar distribution based on $\mathbf{t}$ to have a more accurate estimation.

$\diamond$ Location: The distribution of links over locations where they were observed is given by $\phi_{5 t}$.

$\diamond$ Interaction over time: The distribution of links over 5-min time slices. This plot is helpful to know the number of occurrences of the activity and the number of links when the activity happened.

$\diamond$ Duration: The distribution of time slices where the activity topic $t$ happened over the duration of the activity.

$\diamond$ Number of involved people: Distribution of time slices where the activity topic $t$ happened over the number of people who were involved (having a link assigned to activity topic $t$ in the time slice).

For each discovered activity topic, the annotator gave a relevance score from 1 (not relevant) to 4 (very relevant). The relevance score is a subjective assessment of the annotator about the discovered activity topic, for which we do not have a specific definition for assessing how well the algorithm did for each activity topic. We gave some hints to the annotators by suggesting several dimension to look at: is meaningful? is easy to interpret? corresponds to an actual activity of people? is it a specific activity or it corresponds to multiples activities?. If an activity topic is meaningful, easy to interpret, and corresponds to an actual social activity then ideally it should get the highest score of 4 . If some dimensions is not satisfied then the relevance score is lowered. Note that low relevance score can come from sensing framework (proximity link does not correspond to interaction) and/or the discovery method.

The annotator was also asked to assign each presented activity topic to one or multiple predefined semantic labels. A collaborative annotation session was done online by annotators and authors to decide what are the labels for social activity. From this pilot analysis, we agreed that the set of discovered activity topics can be explained by seven labels:

$\diamond$ dailyNearbyOffice: false positive face-to-face interactions from people who sit very close to each other;

$\diamond$ randomChatWork: work related discussion between people who work together;

$\diamond$ randomChatNonWork: non-work interaction (but not a coffee break) between friends such as chatting, moving together, smoking together;

$\diamond$ coffeeBreak: coffee breaks near a coffee machine or the cafeteria; $\diamond$ restaurant: having lunch in the restaurant area;

$\diamond$ irregularMeeting: meeting of a group of people, which does not always happens at the same time and the same day of the week.

$\diamond$ weeklyMeeting: happening every week at the same time and day.

Note that if the discovered activity topic seems to include multiple activities (for example, randomChatNonWork and CoffeeBreak together), then the annotator can stick multiple labels. All anno- 
tations were done on a shared Google spreadsheet, each set corresponds to one separate sheet. Each annotator was the responsible person of one or multiple sets and need to finish the assigned sets. Furthermore, other annotators can look at his/her annotations and comment. The objective was to find an agreement between annotators rather than averaging annotation from multiple annotators.

Discovered activity topics. Examples of discovered topics are given in Table 1 for which we report the experience of the annotator when she/he look at the visualization of the activity topic. The "Code" field corresponds to the set and the number of the discovered activity topic. The "Observation" field is our text description for the activity topic in terms of who, when, and where. These information can be easily extracted from the activity topic visualization. The "Annotation" field corresponds to the annotated label(s) and relevance score. Finally, we report the annotator comment about the discovered activity topic in italic.

We selected a number of activity topics to demonstrate what kind of activities were discovered and report the justifications from the annotators about their annotations. The topic 3 in set 3 corresponds to coffee break interactions of 13 people in several offices, its visualization is shown in Figure 5. From the plots, we can extract easily that there are this particular activity topic involved 13 people (yellow nodes in the network plot), could happen any time, and took place at the coffee machine. The timeline slot in Figure 5(c) show that this activity happened every working day of the week, and the distributions in Figure 5(d) shows that the events of this activity are usually short (lest than 15 minutes) and that people usually come in group of 2 or 3 people. These findings are summarized in Table 1 and they correspond to the comment from the annotator.

Due to space constraint, we put the visualization of other activity topics in a separated supplementary document, and only report the summarization of these activity topics in Table 1. The topic 30 of set6 is an example of unreal interaction between four people coming from a small office. The annotator can recognize this activity topic as a dailyNearbyOffice since the interaction links occur almost everyday and lasts several hours. The topic 57 in set6 is an example of interaction between friends and between colleagues. The annotator thinks that it is a mix of randomChatWork and randomChatNonWork since these kind of social activity usually involve only 2 or 3 people; and the durations of interaction are usually short (quick chat) but can be relatively long (probably work related discussion). The activity topic 43 of set6 involve 7 people who works together in several projects. Based on the duration and relationship between these people, the annotator labeled it as randomChatWork.

In some case, the discovered activity topic does not correspond to a single activity label. We found that randomChatNonWork is usually confused with randomChatWork since their patterns about number of people, time, and location are very similar. The main difference between the two activity labels lies in the duration of interaction of event and the relationship between people. These information, however, had not been exploited by the model so that the discovered activity topics are not discriminative between the two activity labels. The activity topic 5 of set 6 is a typical example of the confusion between randomChatWork and randomChatNonWork. These are interactions between two people from the same room, which are relatively short but it is unclear if the discussion is work-related. Similarly, the activity topic 57 of set6 is another example of the confusion, but involve more people, and some of them are friends.

The three remaining examples are dedicated to restaurant (set329), irregularMeeting (set6-12), and weeklyMeeting (set4-18). While the patterns of these two examples of restaurant and weeklyMeet-
Table 1: Examples of discovered activity topics, their annotations, and comments from annotators.

\begin{tabular}{|l|l|l|}
\hline Code & Observation & Annotation \\
\hline set4-33 & Who: 13 people & coffeeBreak \\
(Fig. 5) & $\begin{array}{l}\text { When: can be any time, most pop- } \\
\text { ular around 10am and 4pm } \\
\text { relevance: }\end{array}$ \\
\hline
\end{tabular}

"This activity topic corresponds to interactions during coffee breaks; people usually came in group of 2 or 3; lasted around 15 minutes; this activity topic is relevant and it reflects the actual activity."

\begin{tabular}{|l|l|l}
\hline set6-30 & Who: 4 people & dailyNearbyOffice
\end{tabular}

When: can be any time of the day relevance: 1

Where: not available (no connec-

tion to any fixed station)

"Happened almost everyday from 10am to $7 \mathrm{pm}$ with a break at lunch; this activity topic does not seem to correspond to real faceto-face discussion since these 4 people came from a small office and the activity happened almost everyday and lasts several hours. Not a relevant activity."

\begin{tabular}{l|l|l}
\hline set6-43 & Who: 7 people & randomChatWork
\end{tabular}

When: any time, usually after relevance: 4

lunch

Where: not available

"Two or three people discuss together; usually short but can be long up to 2 hours; these people work together thus the topic is very relevant; these seven people come from different offices."

\begin{tabular}{l|l|l}
\hline set6-5 & Who: two people & randomChatWork,
\end{tabular}

When: can be any time of the day randomChatNonWork \begin{tabular}{l|l} 
Where: not available & relevance: 3
\end{tabular}

"Interaction between two colleagues from the same office; usually last less than 15 minutes but it is not clear if the discussion is workrelated since these two people also work together."

\begin{tabular}{l|l|l}
\hline set6-57 & Who: 7 people & randomChatWork,
\end{tabular}

When: can be any time of the day randomChatNonWork

\begin{tabular}{l|l} 
Where: not available & relevance: 3
\end{tabular}

"Irregular interactions involving 2 or 3 people; interaction durations are usually less than 15 minutes but can be up to 2 hours; can be a discussion about work or non-work chatting since some people are friends; The seven people come from different offices."

\begin{tabular}{l|l|l} 
set3-29 & Who: 4 people & restaurant
\end{tabular}

When: between 12:00 and 14:00 relevance: 4

Where: restaurant area

"These 4 people (sitting in the same office) usually come together to have lunch, lasts usually one hour."

\begin{tabular}{l|l|l} 
set6-12 & Who: 8 people & irregularMeeting
\end{tabular}

When: some Mondays and Tues- relevance: 2

days, $5 \mathrm{pm}-6 \mathrm{pm}$

Where: usually at meeting rooms

"It is clear that this activity topic corresponds to one-hour meetings; the 8 people actually work together; the number of attendees vary from 5 to 10, make it hard to interpret the activity topic."

\begin{tabular}{l|l|l}
\hline set4-18 & Who: 11 people & weeklyMeeting
\end{tabular}

When: every Monday at $12 \mathrm{pm}-$ relevance: 4

$1 \mathrm{pm}$

Where: not available

"Group meeting where members are supposed to participate every week; lasting one hour; happened every Monday in the 6 week period." 


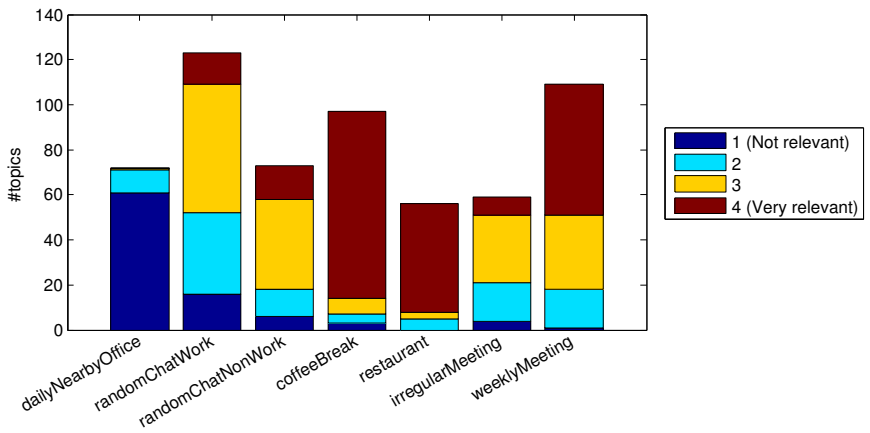

Figure 6: Relevance scores of activity topics in different categories of activity.

ing are clean (with the highest relevance score), the chosen activity topics of irregularMeeting was not easy to interpret for the annotator. More specifically, the activity topics correspond to meetings of one hour, but the number (and the composition) of attendees varied significantly from one meeting to another, making it hard to recognize in which context these meetings were held (for which projects, held by which group of people, etc.)

Subjective evaluation. In overall, $16 \%$ of the activity topics get relevance score 1 (Not relevant), another $16 \%$ of them get score 2 (OK - but not so good), 25\% with score 3 (Good), and the remaining $44 \%$ of activity topics get score 4 (Very relevant). After a close inspection, we found clear patterns on the dependencies between relevance score and the activity label. On one hand, most activity topics with low relevance score were annotated as dailyNearbyOffice, corresponding to groups of people whose desks are very close. While the captured interaction links did not correspond to real interaction due to sensing method (proximity does not always mean interaction), the model was able to regroup them in several activity topics. We see in the next section that there is the possibility to recognize these "unreal" interactions automatically. On the other hand, coffee breaks, lunch in the restaurant, and weekly meetings were well discovered by the model which are relatively easy to interpret. The relevance of the remaining activity topics were dominated by score 3 and 2, corresponding to Good and OK, respectively. As stated earlier in the description of the annotation process, low relevance scores may come from sensing quality and/or the method. Our results confirmed that low relevance scores come mainly from the sensing part (false positive interaction links) while the activity discovery algorithm performs relatively well.

\subsection{Automatic labeling of topic}

In the previous section, we show that many of the discovered activity topics correspond to the actual group activity, and they can be interpreted by people who know the organization well. As the next step, it would be practical to have a system that uncovers multiple activity topics and recognizes automatically their semantic meaning such as weekly meetings or coffee breaks. We formalize the recognition problem in a supervised learning framework where data points correspond to discovered activity topics and the ground-truth is a whether an activity topic correspond to a given label.

As location and social activity are closely related, one may infer the social activity directly from the location of interaction (e.g., having coffee break at the coffee machine). However, location information is not always available, and people may have multiple activities at the same location (e.g., irregular meeting or weekly meeting at the meeting room). We propose to use random forest for labeling discovered activity topics based on various information,
Table 2: Feature extraction for automatic labeling of discovered activity topics.

\begin{tabular}{|l|l|}
\hline Group & Description \\
\hline Location & The distribution of links over locations. \\
Time & $\begin{array}{l}\text { The distributions over time-of-day and day-of-week. } \\
\text { Pumber of active members and distribution of time } \\
\text { People } \\
\text { slices over the number of involved people. }\end{array}$ \\
Duration & $\begin{array}{l}\text { The distribution of time slices over durations. } \\
\text { The distribution of number of links per time slice. }\end{array}$ \\
\hline
\end{tabular}

including location or not. By studying two prediction systems with and without location features, we can see whether it is possible to infer the activity without the need of location data (e.g., for dataset which does not contain location information or the semantic meaning of location is unknown) and how much location information can help improving the prediction performance.

Feature extraction. For the automatic labeling task, each discovered activity topic $t$ is represented by a vector of 49 features belonging to six groups as shown in Table 2. The set of location features consists of the distribution over the file categories of location, which is encoded by $\phi_{5 t}$. The entropy of these multinomial distributions is also used as a feature. Time features come from the distribution over day of the week, $\phi_{3 t}$, and time of the day, $\phi_{h t}$, as well as their entropy. People features are computed based on the detected number of people involved in the activity topic. We used the number of active members (given by GroupUs algorithm) and the distribution over number of attendees (i.e., fraction of active time slices in which there are exactly $X$ people being involved in activity topic $t$, with $X$ varying from 2 to 49), as showed in Figure 5(d,right). Similarly, duration features are computed as the fraction of active time slices of activity topic $t$ which are parts of an activity lasting $Y$ minutes (Figure 5(d,left)). Finally, for link features, we compute the fraction of time slices of the activity topic $t$ which has $Z$ interaction links being assigned to the activity topic.

Automatic labeling performance. The labeling task is to predict if a discovered activity topic corresponds to an activity label. Thus, for the seven activity labels, we have seven binary classification tasks. We use random forest classifier with 1000 trees and all results are obtained with a 10-fold cross-validation process on 492 annotated activity topics.

Table 3 reports automatic labeling accuracies for the seven predefined activity labels. We use majority class predictor as a baseline and compare its performances with two random forest systems: one with location features and one without location features. As can be seen, all activity labels are predictable to some degrees. coffeeBreak and restaurant can be reliably recognized by random forest systems with and without location features. On the contrary, the automatic recognition systems do not outperform the baseline performance for randomChatNonWork. This issue may come from the fact that it not easy to distinguish between randomChatNonWork and randomChatWork without knowing the actual relationship between people (e.g., close friends, collaborators of same project). This assumption is confirmed by the prediction results of the special activity label random Chat which merges random ChatWork and randomChatNonWork. The reduction in the number of errors (between RF and Baseline) for randomChatNonWork, randomChatWork, and random Chat are respectively $0 \%, 32 \%$ and $47 \%$.

Comparing the two random forest systems with and without location features, we found that their performances are comparable for many activity labels except randomChat and coffeeBreak. To verify if the location information is key to distinguish these two 
Table 3: Automatic labeling accuracies of random forest systems with and without location features. Baseline performance was computed based on majority class predictor.

\begin{tabular}{|l|l|l|l|}
\hline Category & Baseline & RF & RF w/o location \\
\hline dailyNearbyOffice & 0.86 & 0.90 & 0.89 \\
randomChatWork & 0.75 & 0.83 & 0.81 \\
randomChatNonWork & 0.85 & 0.85 & 0.85 \\
coffeeBreak & 0.80 & 0.99 & 0.95 \\
restaurant & 0.99 & 0.99 & 0.98 \\
irregularMeeting & 0.88 & 0.89 & 0.88 \\
weeklyMeeting & 0.78 & 0.88 & 0.88 \\
\hline randomChat & 0.70 & 0.84 & 0.79 \\
randomChatCoffee & 0.52 & 0.85 & 0.86 \\
\hline
\end{tabular}

categories, we apply merging technique again to have a new label called randomChatCoffee. Not surprisingly, the location information is not helpful to distinguish activity topics of the merged category random ChatCoffee and the rest. At the end, location information is helpful to distinguish between randomChat and coffeeBreak, while the prediction performance for other activity categories does not depend significantly on location information.

\section{CONCLUSION}

This study contributes to the understanding of social activity and opens the possibility to infer social activity from interaction between people. We have proposed a variant of topic model which can discover various activity topics from timestamped, localized social interactions being sensed by BT and IR sensors. We also showed how to assign these discovered topics to common sense activity label, thus enabling a fully automatic framework for sensing and inferring social activity based on mobile sensor network. The analysis was conducted on a real interaction data set in an organization, and the method was validated by their people. The subjective evaluation results suggest that many discovered activity topic are highly relevant, while false positive interactions were not filtered completely from the data set. Interestingly, these sensing noise were regrouped in several activity topics, thus the noise may be filtered by the proposed probabilistic method. Finally, our experiment on the automatic labeling demonstrates that some social activity can be recognized reliably by social interaction, even when the location of the interaction is not available.

As future work, we are interested in applying the framework to human behavior analysis to characterize the relationship between social activity and personal state such as mood or job performance. Our framework can be extended to incorporate more behavioral features such as speech or body activity. While this study considers social network sensed by multiple subjects, some of the ideas can be applied to interaction data being sensed by a single individual. Another direction that we want to investigate is to compare our global network analysis with ego network analysis to understand the advantages and shortcomings of each approach.

\section{Acknowledgments}

This research was funded by SNSF SONVB project.

\section{REFERENCES}

[1] N. Aharony, W. Pan, C. Ip, I. Khayal, and A. Pentland. Social fmri: Investigating and shaping social mechanisms in the real world. Pervasive and Mobile Computing, 7(6):643-659, 2011.

[2] R. Albert, H. Jeong, and A.-L. Barabási. Internet: Diameter of the world-wide web. Nature, 401(6749):130-131, 1999.
[3] L. Backstrom, E. Sun, and C. Marlow. Find me if you can: improving geographical prediction with social and spatial proximity. In Proc. $W W W$, pages 61-70, 2010.

[4] L. Bao and S. S. Intille. Activity recognition from user-annotated acceleration data. In Pervasive Computing, pages 1-17. Springer, 2004.

[5] D. M. Blei, A. Y. Ng, and M. I. Jordan. Latent dirichlet allocation. the Journal of machine Learning research, 3:993-1022, 2003.

[6] A. T. Campbell, S. B. Eisenman, N. D. Lane, E. Miluzzo, and R. A. Peterson. People-centric urban sensing. In Proc. WICON, page 18, 2006.

[7] C. Cattuto, W. Van den Broeck, A. Barrat, V. Colizza, J.-F. Pinton, and A. Vespignani. Dynamics of person-to-person interactions from distributed rfid sensor networks. PloS one, 5(7):e11596, 2010.

[8] X. Chen and J. Yang. Towards monitoring human activities using an omnidirectional camera. In Proc. ICMI, pages 423-428, 2002.

[9] M. Cristani, R. Raghavendra, A. Del Bue, and V. Murino. Human behavior analysis in video surveillance: a social signal processing perspective. Neurocomputing, 2012.

[10] T. M. T. Do and D. Gatica-Perez. Contextual grouping: discovering real-life interaction types from longitudinal bluetooth data. In Proc. MDM, volume 1, pages 256-265, 2011.

[11] T. M. T. Do and D. Gatica-Perez. Human interaction discovery in smartphone proximity networks. Personal and Ubiquitous Computing, 17(3):413-431, 2013.

[12] W. Dong, B. Lepri, and A. S. Pentland. Modeling the co-evolution of behaviors and social relationships using mobile phone data. In Proc. MUM, pages 134-143, 2011.

[13] N. Eagle and A. Pentland. Reality mining: sensing complex social systems. Personal and ubiquitous computing, 10(4):255-268, 2006.

[14] S. A. Golder and M. W. Macy. Diurnal and seasonal mood vary with work, sleep, and daylength across diverse cultures. Science, 333(6051):1878-1881, 2011.

[15] M. C. Gonzalez, C. A. Hidalgo, and A.-L. Barabasi. Understanding individual human mobility patterns. Nature, 453(7196):779-782, 2008.

[16] C. A. Hidalgo and C. Rodriguez-Sickert. The dynamics of a mobile phone network. Physica A: Statistical Mechanics and its Applications, 387(12):3017-3024, 2008.

[17] B. Lavelle, D. Byrne, G. J. Jones, and A. F. Smeaton. Bluetooth friendly names: bringing classic hci questions into the mobile space. In Proceedings of the 21 st British HCI Group Annual Conference on People and Computers: HCI... but not as we know it-Volume 2, pages 63-66. British Computer Society, 2007.

[18] B. Lepri, J. Staiano, G. Rigato, K. Kalimeri, A. Finnerty, F. Pianesi, N. Sebe, and A. Pentland. The sociometric badges corpus: A multilevel behavioral dataset for social behavior in complex organizations. In SocialCom/PASSAT, pages 623-628, 2012.

[19] C.-H. Lu and L.-C. Fu. Robust location-aware activity recognition using wireless sensor network in an attentive home. Automation Science and Engineering, IEEE Transactions on, 6(4):598-609, 2009.

[20] A. Madan, M. Cebrian, D. Lazer, and A. Pentland. Social sensing for epidemiological behavior change. In Proc. 
UbiComp, pages 291-300, 2010.

[21] A. Noulas, S. Scellato, C. Mascolo, and M. Pontil. An empirical study of geographic user activity patterns in foursquare. ICWSMâÁŹ11, 2011.

[22] D. O. Olguin, P. A. Gloor, and A. S. Pentland. Capturing individual and group behavior with wearable sensors. Proc. AAAI Spring Symposium, 9, 2009.

[23] D. Olguin-Olguin, B. N. Waber, T. Kim, A. Mohan, K. Ara, and A. Pentland. Sensible organizations: technology and methodology for automatically measuring organizational behavior. Trans. Sys. Man Cyber. Part B, 39(1):43-55, Feb. 2009.

[24] S. Phithakkitnukoon, T. Horanont, G. Di Lorenzo, R. Shibasaki, and C. Ratti. Activity-aware map: Identifying human daily activity pattern using mobile phone data. In Human Behavior Understanding, pages 14-25. Springer, 2010.

[25] Y. Song, L.-P. Morency, and R. Davis. Multimodal human behavior analysis: learning correlation and interaction across modalities. In Proc. ICMI, pages 27-30, 2012.

[26] A. Vahdatpour, N. Amini, and M. Sarrafzadeh. Toward unsupervised activity discovery using multi-dimensional motif detection in time series. In Proc. IJCAI, pages 1261-1266. Morgan Kaufmann Publishers Inc., 2009. 